\title{
SULLA ELETTRIZZAZIONE DEL MERCURIO DETTA PER STROFINIO.
}

\author{
Nota $I$ di ELIGIO PERUCCA.
}

\section{\& 1. - Introduzione.}

Scopo di questo lavoro è di rendere noto un fenomeno presentato dal mercurio nelle condizioni ordinarie di esperienze del genere, ciò̀ nell' aria a pressione normale e a temperature ordinarie $\left(\mathbf{1 0}^{0}-\mathbf{4 0 ^ { \circ }}\right)$. Questo fenomeno, sfuggito alla osservazione dei precedenti sperimentatori, è il seguente: una superficie di mercurio appena formata si elettrizza energicamente di segno positivo se posta a contatto con quarzo, vetro, paraffina, ceralacca, ebanite, ceva vergine, colofonia, lana, ecc. "), ma, in un tempo variabile da pochissimi secondi fino a qualche ora, la superficie di mercurio va perdendo la sua eccitabilità positiva, passa per un istantc di eccitabililà nulla, per poi acquistare eccitabilitì negativa, la quale cresce asintoticamente fino a un valore limite.

Vien subito in mente di connettere questo fenomeno con le variazioni col tempo della costante capillare del mercurio, osservate già da molti anni ${ }^{2}$ ), e con le variazioni col tempo del comportamento ottico nella riflessione su superfici di mercurio ${ }^{3}$ ).

2) Coordinando i miei risultati con quelli di P. E. Shaw ( Proc. Roy. Soc. A. 94, p. 16, 1917; dal titolo si prevede la pubblicazione di alteriori risultati sull'argomento, ma non mi risulta che ciò sia stato fatto fino ad ora), il fenomeno presumibilmente avviene al contatto del mereurio con tutti i corpi elencati da tale autore, esclusi celluloide $\theta$ caucciù da un lato e amianto dall'altro. $v$. però la nota (') a p. 38.

2) P. es. A. Kahliine, An. der. Phys. 7, p. 471, 1902.

') J. J. Haak e K. Sissingh, K. Ak. Amst. Pr., 21, p. 678, 1919. Sarebbe stato per me interessantissimo porre in relazione $i$ risultati di questo recente lavoro con le mie osservazioni ; ma ciò non mi è stato possibile, pcrchè finora non ho potuto procurarmi il lavoro originale, 
Il fenomeno in questione permette di interpretare i risultati numerosi ottemuti da vari sperimentatori sulla elettrizzazione del urercurio per strofinio.

Mi limiterò in questa nota a :

$1 .^{0}$ descrivere le condizioni nelle quali ho eseguito le esperienze ed esporne i risultati,

$2 .^{0}$ coordinare i risultati ottenuti dagli altri sperimentatori.

Rinvio ad un'altra comunicazione la discussione sulla causa del fenomeno.

La superficie di mercurio di fresco preparata evidenteinente si ra modificando. In base alle esperienze già eseguite, risulta necessario il contatto della superficie di mercurio con l'aria.

\section{$\$ 2$. Condizioni sperimentali.}

Veniva preparata in un piattello di circa $8 \mathrm{~cm}$. di lato una superficie fresca di mercurio, filtrando questo metallo attraverso carta bibula forellata, oppure facendolo effluire da un imbuto a collo affilato. Il mercurio era stato preventivamente purificato con acido cloridrico e acido nitrico, lavato in acqua distillata, poi asciugato.

Ho usato piattelli di ferro, di alluminio, di vetro. Il piattello di ferro è preferibile, perchè non viene attaccato dal mercurio, come succede per l'alluminio, e non dà segno di elettrizzazione per strofinio col mercurio, come succede pel vetro. Ma l'esperienza riesce ugualmente in tutti e tre i casi. Ho anche usato due recipienti di ferro cilindrici, l' uno di circa $3 \mathrm{~cm}$. di diametro, l'altro, invece, molto più ampio.

del quale mi sono note solo le recensioni dei Sc. Abstracts e dei Beiblätter.

Ad esempio, anche L. P. Wheeler (Phil. Mag., 22, p. 229, 1911) osserva che una superficie tersa di merourio perde rapidamente la su a fresshezza. 
In molte esperienze ') il mercurio era in commitcazionepermanente, mediante un filo di rame, con an elettrometro a foglia di alluminio. Trattandosi per ora di esperienze lul- $^{\prime \prime}$ ramente qualitative, dirò solo che ogni divisione della scala corrispondeva, in media, a circa 10 volts. Poggiavo delicatamente sul mercurio il disco isolante per circa due secondi, poi lo allontanavo.

Ma la maggior parte delle esperienze sono state fatte tenendo il mercurio a terra poggiandovi su delicatamente il disco dell' isolante per circa due secondi, poi introdncendo quest'ultimo in un pozzo di Faraday connesso all'elettrometro a foglia d'alluminio.

Determinavo il segno del potenziale e quindi della carica indicata dall' elettrometro avvicinando al mercurio - nella prima disposizione - o al pozzo di Faraday - nella seconda - una bacchetta di ebanite strofinata con lana. Numerose prove eseguite con una pila Zamboni mi hanno assicurato che in ogni caso la mia ebanite si caricava negativamente.

Per il mio scopo, risultò indifferente, come del resto è naturale, l' usare l' una o l' altra disposizione.

Gli isolanti con i quali toccavo il mercurio sono stati :

Quarzo (lamina perpendicolare all'asse con facce piane e levigate). Mi sono più volte assicurato che non si producessero fenomeni piezo- o piroelettrici, ma che l' elettrizzazione si manifestasse nel quarzo pel solo effetto del contatto con il mercurio.

Vetro (due dischi a facce piane e levigate di cristallo di qualità diversa; e parecchi tubi di vetro di circa $2 \mathrm{~cm}$. di diametro di qnalità diverse, tubi destinati, in particolare,

1) Le esperienze eseguite sono state parechie centinaia, distribuite in circa 70 giorni. Quasi sempre nua esperienza consisteva in numerose prove sncessivamente eseguite. Ogni risultato è stato verificato con numerose esperienze. 
a essere introdotti nel mercurio nei recipienti di ferro cilindrici di cui ho parlato precedentemente.

Alcune prove mi hanuo assicurato che il fenomeno si svolge in modo analogo anche quando $Y$ isolante sia:

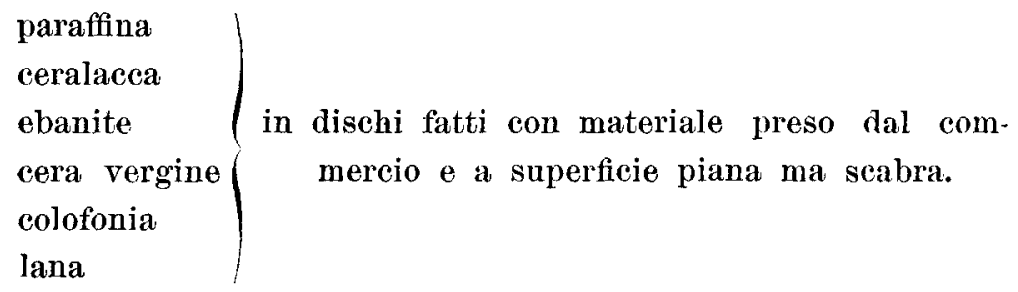

Preferisco però limitarmi a considerare le sole esperienze col vetro e con il quarzo, e specialmente quelle col quarzo, il solo, tra gli jsolanti solidi che avevo a disposizione, chimicamente definito ').

I dischi di lana erano incollati a un disco di paraffina o ebanite, sul quale era innestato un manico metallico, con cui sostenevo il disco. Così il disco era ben isolato, ma era evitata la produzione di elettricità che si manifesta sfiegando con le dita un isolante.

Una prova ordinaria consisteva nel sovraporre delicatamente al mercurio il disco di materiale isolante, lasciarvelo a contatto circa due secondi, poi asportarlo e misurare la carica del mercurio $\left(1 .^{2}\right.$ disposizione) o dell' isolante $\left(2 .^{\mathrm{a}} \mathrm{di}\right.$ sposizione).

L' elettrizzazione che così acquistano mercurio e isolante è fortissima; l'elettrometro indica sia nell'una che nell'altra delle disposizioni su indicate, aventi infatti capacità elettro-

1) Lo zolfo, sia poggiato che immerso bruscamente nel mercurio, si ¿ sempre elettrizzato positivamente; più fortemente, perd, quando sia poggiato. E probabile che, con una sufficiente velocità di immersione, anche lo zolfo si elettrizzi negativamente.

Non credo sia opportuno l'uso dello zolfo, chè, anche a temperatura ordinaria, reagisce col mercurio.

Quarzo destrogiro o levogiro, perpendicolare all'asse o parallelo all'asse danno luogo al fenomeno in esame, in egual roodo. 
statiche dello stesso ordine, un potenziale che raggiunge anche parechie centinaia di volts.

Dopo ogni prova, scaricavo il mercurio ponendo al suolo il piattello mediante la mano; scaricavo in pochi secondi l' isolante ponendolo lateralmente ad alcuni cm. da una fiammella a gas tenuta in una stanza attigua a quella delle esperienze.

Le esperienze sono state eseguite con superfici terse di mercurio e di isolante.

Ho già detto che finora ho eseguite le esperienze nell'aria a pressione e temperatura ordinarie. Noterò soltanto che anche in queste esperienze preliminari potevo far variare entro limiti abbastanza estesi e conoscinti l'umidita relativa, ma anche la temperatura dell' aria sovrastante al mercurio: ficevo quasi contemporaneamente le esperienze nelle parti piǹ fredde del laboratorio $\left(10^{\circ}\right.$ circa $)$ e sul termosifone $\left(40^{\circ}\right.$ circa).

\section{3. - Esperienze ordinarie e loro risultati.}

Mercurio in un piattello, toccato con la superficie del disco di quarzo. Classifico i risultati secondo il modo col quale sono stati ottenuti, ma, come si vedrà dal seguito del lavoro, non credo si debba attribuire all' umidità l'importanza che da questa classificazione scaturisce:

a) in ambiente freddo e umido (nelle parti fredde del laboratorio, con tempo nebbioso o pioggia). Soltanto dopo parecchie filtrazioni attraverso carta bibula ben secca riescivo ad ottenere il fenomeno da me indicato. E, quando l'ottenevo, la variazione di segno della eccitabilità del mercurio avveniva in pochissimi secondi. Viene quindi il dubbio che le numerose esperienze con risultato negativo che si hanno. in queste condizioni siano dovute alla rapidità con la quale il fenomeno avviene, o addirittura al non formarsi, durante la filtrazione, della superficie fresca di mercurio, sulla quale il fenomeno si presenta. 
b) in ambiente piuttosto secco (con bel tempo anche nelle parti fredde del laboratorio, ma ordinariamente ad un paio di metri dal termosifone, a una temperatura di circa $18^{\circ}$ ). Il fenomeno si produce quasi sempre dopo una sola filtrazione, pur di nsare carta da filtro ben pulita e asciutta; asso richiede qualche minnto per svolgersi.

Esempio: Eccitabilitì del quarzo posto a contatto con superficie di merenrio vecchia di dne giorni e misurata dalla deviazione all' elettrometro connesso col pozzo di Faraday seconda delle disposizioni su accenuate): +18 .

Eccitabilità del quarzo a contatto con lo stesso melcurio la cui superficie è stata appena formata mediante filtrazione :

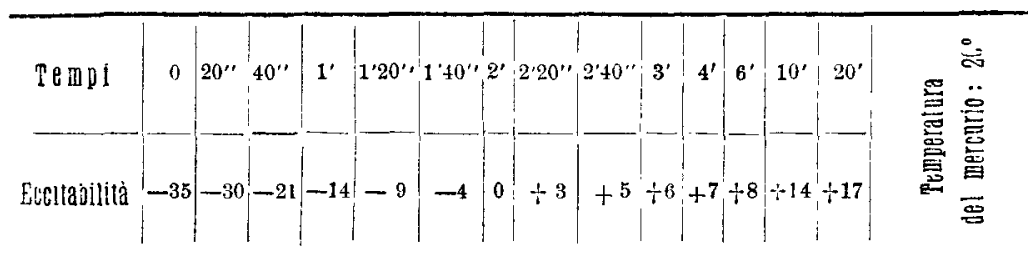

Avverto che a questi numeri non si deve attribuire alcun valore quantitativo; basti notare, infatti, che l' eccitabilitì rel quarzo così misurata mediante il potenziale indicato dali' elettrometro, eresce nei primi istanti con la durata del contatto tra quarzo e mercurio. Ciò è probabilmente dovuto a una lieve condncibilità del quarzo.

I pochi casi nei quali il fenoweno nou si è prodotto si possono attribuire con grande verosimiglianza a qualche variazione accidentale $\mathrm{e}$ non facilmente verificabile nelle condizioni dell'ambiente o della filtrazione del mercurio.

c) ambiente caldo e secco. Il fenomeno si produce senza bisogno di speciali precanzioni nel filtrare il mercurio, ma questa volta esso richiede molti minuti per svolgersi. Con il mercurio già caldo, filtrato e tenuto sul termosifone, a circa $40^{\prime \prime}$, l'eccitabilità iniziale positiva del mercurio cambiava segno sol dopo una, due, tre ore. 


\section{\$4. - Interpretazione del fenomeno.}

L'esistenza di questi fatti, verificati in varie centinaia di superficie di mercurio al loro contatto con una stessa superficie di quarzo, induce a pensare che la superficie fresca di mercurio abbia proprietà fisiche nettamente distinte da quelle di una superficie vecchia.

Ho gia detto che, per altre vie, vari sperimentatori erano già stati condotti a questo risultato.

II mercurio entra dunque nel numeroso gruppo dei metalli che si elettrizzano di segno diverso secondo lo stato della loro superficie o il modo con cui vengono strofinati. Si tratta, veramente, di risultatj tra loro slegati e non sempre concordi : così De Ia Rive ${ }^{1}$ ) attribuisce all' umiditì una grande importanza; J. A. Me Clelland e C. J. Power ${ }^{3}$ ) non confermano il fatto.

Il caso del mercurio è più semplice, perchè si può preparare in pochi secondj una superficie mova di mercurio in condizioni sensibilmente eguali. Inoltre l' elettrizzozione dי] mercurio si ottiene col semplice contatto col dielettrico; quindi sono evitate tutte le complicazioni dello strofinio, in particolare l'asportazione reciproca di particelle e il riscaldamento. Avviene però un rimescolamento del mercnrlo, in seguito al distacco dell'isolante.

ì certo che la superficie fresca del mercurio va alterandosi col tempo, quando sia in presenza dell' aria (v. $§ 5$ ). Rinvio alla nota seconda la discussione sulla causa del fatto. Dai risultati esposti nel paragrafo precedente sembrerebbe naturale attribuire il fenomeno ad adsorbimento di vapor d'acqua da parte della superficie di mercurio; ma nelle condizioni b) e c), il cambiamento di segno nella eccitabilità

1) Pogg. Ann., 37, p. 506, 1836.

2) Roy. Irish. Ac. Proc., A. 34, p. 40, 1918; mi sono note solo lo receusioni dolla Rev. Scientifique, e dell' Elettricista. Altro lavoro recente B dovuto a W. M. Jones (Phil. Mag., 29, p. 261, 1915). 
del mercurio non viene accelerato dall' alitare sul mercurio fino a formarvi su una pellicola di rugiada che rapidamente sparisce. Nè il cambiamento di segno viene ottenuto rapidamente preparando la superficie fresca di mercurio in ambiente artificialmente saturato di vapor d'acqua e tenendovela alcuni minuti.

Esperienze ulteriori, fatte in un ambiente ove siano note e variabili a piacere la natura e le condizioni fisiche del gas sovrastante al mercurio, nii permetterano, io spero, di trovare la causa del fenomeno.

\section{5. - Esperienze speciali e loro risultati.}

Ho eseguito varie esperienze per assicurarmi che il fenomeno descritto sia dovuto all' esistenza sul mercurio di due specie di superfici, l' una fresca, che al contatto con vetro, quarzo, ecc., si carica positivamente; l'altra vecchia, ottenuta per un'alterazione non ancor ben definita della precedente, la quale si carica negativamente al contatto con questi isolanti.

Esporrò solo le seguenti esperienze, per l'applicazione che ne farò nel paragrafo seguente:

a) Ho fatto numerose volte esperienze contemporanee con la stessa superficie di mercurio e diverse superfici di isolante, nel mio caso il disco di quarzo e un disco di vetro da specchi.

Il fenomeno del cambiamento di segno avviene sensibilmente allo stesso istante sia pel quarzo che pel vetro, forse un po' prima pel quarzo ').

Ho fatto esperienze contemporaneamente con una superficie vechia di mercurio e una superficie fresca di mercurio,

1) Posso accennare che esperienze analoghe con la paraffina, l' ebanito e la ceralacca hanno mostrato che il cambiamento di segno dell'eccitabilita del mercurio si ha prima nel quarzo e vetro, poi nella ceralacca e nella paraffina quasi contemporaneamente, intine nell' ebanite. 
toccate con la stessa lamina di quarzo. Così mi sono assicurato che, mentre il fenomeno avviene nella superficie fresca di mercurio, la lamina di quarzo dimostra ai suoi contatti con la superficie vecchia di conservare sempre le stesse proprietà.

In tutte le prove occorrenti per queste esperienze non ho mai toccato la superficie del dielettrico altro che col mercurio.

Nelle ultime esperienze ho sempre verificato, mediante una superficie di mercurio vecchia di molti giorni, quale fosse la condizione di eccitabilità dell' isolante ').

Pur dovendosi ammettere l'influenza dello stato superficiale del quarzo o del vetro ${ }^{2}$ ), è logico concludere che il fenomeno arviene alla superficie del mercurio e non alla superficie del dielettrico.

Ho fatto anche prove per cercare un' influenza dello stato o della storia precedente della superficie del dielettrico, ma i fatti osservati sono irregolari e non mi è riuscito di riprodurli a mia volontà. Essi si notano nel vetro e nel quarzo, e si riducono ordinariamente a una variazione del valore dell'eccitabilità, non del segno di questa.

Vetro e quarzo, lasciati all'aria libera qualehe tempo, diventano poco eccitabili al contatto col mercurio, ma ritornano alle condizioni ordinarie mediante una semplice pulitura con un qualunque corpo asciutto.

Rarissime volte mi è riuscito come di ritardare il momento in cui una superficie fresca di mercurio cambia il segno della sua eccitabilità, pulendo rapidamente il dielettrico con cotone ben secco, oppure scaldando la superficie del dielet. trico a temperatura molto elevata $\left(200^{\circ}-300^{\circ}\right)$, ma aspettandone il raffreddamento prima di fare la prova ${ }^{3}$ ).

1) Di molti giorni, intendesi qui, tre o quattro giorni, successivamente (v. Nota $I^{2}$ ) ho usato un modo molto migliore per la verifica in questione.

3) V. in particolare $i$ lavori di Christiansen e di Shaw.

3) Vedi però, l' esito delle esperienze fatte successivamente, e descritte nella Nota II . 
b) Un recipiente di ferro cilindrico, profondo circa $12 \mathrm{~cm}$, diametro $3 \mathrm{~cm}$. circa, viene riempito di mercurio per circa $4 \mathrm{~cm}$. In questo mercurio posso immergere un tubo di vetro chinso a un' estremità (p. es. un tubo da saggio). Le cose sono disposte in modo che, introducendo il tubo, il mercurio sale fino al bordo del recipiente di ferro. Si ha così modo di porre a contatto del tubo di vetro una superficie di mercurio costretta a distendersi grandemente e, quindi, paragonabile, con ogni probabilità, a una superficie di mercurio preparata di fresco.

Versato nel recipiente di ferro un po' di mercurio a superficie vecchia, lo tocco con l'estremità del tubo di vetro, senza produrre grande estensione della superficie di mercurio. Verifico che l'estremità del tubo è carica positivamente.

Immergo, sia pur dolcemente (cioè in $3-4$ secondi), il tubo di vetro fino in foncto al cilindro, in modo che la superficie di mercurio si distenda e venga a lambire per quasi $12 \mathrm{~cm}$. il tubo di vetro. Ora il tubo di vetro risulta fortemente carico di elettricità negativa.

Parecchie volte, con un tubo di vetro bene ascintto e, quindi, pochissimo conduttore, ho potuto addirittura constatare che l' estremità del tubo era carica positivanente, le zone successive erano cariche negativamente.

c) Ritento le esperienze nelle stesse condizioni di $b$ ), solo sostituendo allo stretto cilindro di ferro un recipiente di ferro di parecchi $\mathrm{cm}$. di diametro - nel mio caso una vaschetta di $20 \mathrm{~cm}$. di diametro e $6-7 \mathrm{~cm}$. di profondità.

Le esperienze precedenti riescono soltanto se il tubo di vetro viene introdotto nel mercurio molto vivacemente, quasi vi venga battuto; anche così alcune volte non si riesce a ottenere il vetro carico negativamente, ma soltanto una elettrizzazione positiva del vetro più debole di quella che si ottiene con una immersione delicata. Non è esuluso che con velocità di immersione sufficientemente alte il fenomeno si produca anche in questo caso. 
Queste esperienze si accordano immediatamente con l'interpretazione esposta al $\S 4$.

d) Una superficie vecchia di mercurio acquista la caratteristica di superficie fresca, cioè si elettrizza positiva mente, se il mercurio a cui essa appartiene viene sbattuto o rimescolato vivacemente. Probabilmente la filtrazione agisce in questo senso.

Si può attribuire a rimescolamento l' effetto della temperatura notata a pag. 40, e ciò̀ la lentezza con cui avviene il fenomeno nel caso del $\S 3, c)$.

e) Una superficie fresca di mercurio veniva coperta ermeticamente con una lastra di vetro, così da toglierla dal contatto dell' aria. Una tal superficie rimaneva fresca per moltissime ore (fino a 24, nelle mie prove), mentre una superficie preparata in modo eguale, dello stesso mercurio, ma all'aria, dava luogo al fenomeno in circa mezz' ora.

Questa prova, ripetuta numerosissime volte, obbliga a considerare l'aria come causa essenziale del fenomeno.

f) Infine, facendo gocciolare il mercurio ') sulle superfici isolanti di quarzo, vetro, colofonia, cera vergine, lana, ebanite, ceralacca, parafina, queste risultarono sempre cariche negativamente; il mercurio risultò carico positivamente.

Tutti questi isolanti, posti a contatto con la superficie vecchia dello stesso mercurio, si elettrizzavano positivamente. Anche questo fatto si accorda facilmente con l'ipotesi del $\S 4$, ma noterò che questo non è un modo semplice di eseguire le esperienze, perchè si sostituisce al semplice contatto l'urto mal definito e accompagnato dallo sminnzzamento delle goccie di mercurio.

1) Esperienze preliminari mi assicuravano che le goceioline di mercurio non erano elettrizzate prima di battere sull' isolante. Non mi consta che il Christinnsen abbia esteso al mercurio le sue esperienze di ballo-elettricità. 


\section{\$ 6. - I risultati dei precedenti sperimentatori.}

Sono numerosi, ma disparati. Disgraziatamente, gli autori raramente indicano con sufficiente dettaglio le condizioni delle esperienze. Invero, non risulta, p. es., che alcuno abbia pensato finora che occorresse indicare la larghezza del recipiente contenente il mercurio rispetto alla grossezza della bacchetta isolante che vi si immerge. Per questo, è difficile verificare e trovare una spiegazione per i risultati ottenuti. Ma, limitandomi all'elenco dei fatti più importanti, sarà facile accorgersi che tutti - tranne uno - si possono far dipendere dal fenomeno da me descritto.

Canton e Le Roy trovano risultati discordanti per l'elettrizzazione del mercurio a contatto col vetro e con qualche altro isolante; l' uno trova il mercurio negativo, l' altro positivo.

Ingenhousz (1784) tenta accordare questi risultati, attribuendoli a diversa velocità di tocco dell' isolante col mercurio. Le sue ricerche lo conducono al risultato che il mercurio si elettrizza positivamente per un tocco lento con vetro, canfora, lacca, caucciù, negativamente per un tocco rapido.

$\grave{E}$ questo $i l$ solo dei risultati finora ottenuti sull'elettrizzazione del mercurio, che è in disaccordo netto con le esperienze da me eseguite.

Ma tutti gli sperimentatori che dopo Ingenhousz hanno rifatto le esperienze sull'argomento (Dessaignes, Riess, Shaw) hanno trovato risultati opposti a quelli dati da Ingenhonsz.

Dessaignes ${ }^{1}$ ) è autore del lavoro più dettagliato sull'elettrizzazione del mercurio per contatto con isolanti. Per quanto si tratti di un lavoro condotto in un modo strano, i suoi risultati fondamentali sono benissimo interpretabili dal mio punto di vista.

1) Ann. de Chim. et de Phys., 2, p. 59, 1816. 
Eccoli : col barometro alto (quindi, probabilmente, con tempo seceo) il mercurio tende ad elettrizzarsi positivanente; col barometro basso (quindi, probabilmente, con tempo umido) il mercurio tende ad elettrizzarsi negativamente; sembra quindi che il Dessaignes si sia trovato in condizioni analoghe a quelle del $\$ 3, a$ ) e c); che sia proprio l'umidità relativa a produrre tale diversità di risultati, è cosa dubbia, specialmente dopo le prove di pag 42), primo capoverso;

il mercurio che è toccato da ceralacca, vetro, zolfo ${ }^{1}$ ) si elettrizza negativauente; si elettrizza positivamente quando sia da essi battuto; con una certa velocità di tocco, il mercurio sembra ineccitabile.

Ma uno dei risultati del Dessaignes è inesatto cortamente: vetro freddo in mercurio caldo non si elettrizza; vetro caldo in mercurio freddo si elettrizza fortemente. Di un altro r.isultato, quello riguardante l'influenza di una stretta fasciatura nella parte del corpo isolante non introdotta nel inercurio, non sono riuscito ad avere conferma.

Riess ${ }^{2}$ ) asserisce che il mercurio pulito a contatto con vetro pulito si elettrizza negativamente. Questo risultato può benissimo coesistere con i miei. Dice anche che la velocità del tocco tra isolante e mercurio non ha influenza: io credo di poterne desumere che egli si sia trovato nel caso del $\S 5$, c) (secondo capoverso).

Infine, in un recente lavoro sull' elettrizzazione per strofinio, P. E. Shaw ${ }^{3}$ ) ha preso in considerazione il comportamento del mercurio; disgraziatamente sorvola su varie delle condizioni sperimentali. Forse per questo non $\mathrm{mi}$ è stato possibile riprodurre tutti i fenomeni descritti da questo antore. P'ur prescindendo dalla connessione tra l'interpretazione da me proposta e $\mathrm{i}$ fenomeni di capillarità e ottici già accennati, io noterò che il fenomeno permette di spiegare

1) Y. nota a pag. 38.

3) Reibungselektrizität, vol. II, p. 362 e segg.

s) Lav. eit., p. 25. 
nel modo più semplice i risultati del Shaw da me verificati, senza introdmre il concetto di un' « anormalità » alla superficie del dielettrico, prodotta dall' urto di questo col mercurio. Ton sono, naturalmente, in grado di discutere i risultati indicati dal Shaw e da me non saputi ritrovare.

Nelle esperienze a temperatura ordinaria, il Shaw trova che bacchette di tutti i dielettrici - eccetto celluloide e cancciù da un lato, amianto dall' altro lato - introdotte gentilmente nel mercurio, lo elettrizzano negativamente; introdotte vivacemente, lo elettrizzano positivamente. is il fonomeno già scoperto dal Dessaignes, corrispondente a quello del $\S 5$ b) e c). Potrei notare nuovamente che con lo zolfo nè l'esperienza $\S 5$ b) nè l'esperienza $\$ 5 f$ ) mi sono mai riuscite; lo zolfo si è elettrizzato sempre positivamente, sia pur più debolmente quando veniva immerso bruscamente nel mercurio.

Shaw trova poi che vetro, mica e altri dielettrici «anormali » (perchè riscaldati alla « temperatura critica») elettrizzano positivamente il mercurio per contatto sia gentile che violento.

Se il mercurio si elettrizza positivamente al contatto delicato, è evidente dal mio punto di vista che al tocco brusco il mercurio resta positivo. Limitandomi al caso del contatto delicato, dirò ora soltanto che l'anormalità prodotta dal riscaldamento spariva così rapidamente nelle mie esperienze, che non ne rimanera traccia quando il dielettrico era tornato freddo. Percio fui dapprima ') indotto a negare l' esistenza dell'anormalità, alla quale il Shaw attribuiva una notevole stabilità. Più tardi, ottenuto tale stato anormale, ne no potuto studiare alcune caratteristiche (v. nota II).

E probabile che l' «anormalità 》 dovuta al riscaldamento potrebbe consistere anche in una semplice modificazione dell'atmosfera superficiale del dielettrico, modificazione che av-

1) Atti Acc. Tor., 55, p. 452, 1920. 
viene in grado sufficiente solo a una certa temperatura (temperatura critica). (V. pag. 43 , in fondo).

Infine, secondo Shaw, una bacchetta di vetro, immersa nel mercurio, si carica fortemente con carica + ; ripetendo la prova molte volte, la carica + finisce col diventare molto piccola; ma la eccitabilità iniziale è ristabilita se si frega il il vetro con la mano o con un batuffolo di cotone.

Non mi ̀̀ stato facile verificare neanche questo risultato del Shaw. Parechie volte ho provato a poggiare gentilmente fino a più di 100 volte di segnito il quarzo e il vetro sul mercurio; non ho trovato notevole differenza di eccitabilità. Se le successive immersioni si fanno un po' rapidamente, si ha l'effetto indicato dal Shaw, ma si ricade nel fenomeno del $\S 5 \quad d$ ); ciò si accorda col fatto che il quarzo e il vetro, i quali dopo qualche decina di rapide immersioni si mostravano debclmente carichi, riassumevano immediatamente o, al massimo, dopo pocbi secondi, l'eccitabilità ordinaria se posti a dolce contatto col mercurio per il solito tempo di due secondi.

Ancora in vari altri casi ho trovato l'effetto indicato dal Shaw, e precisamente quando, per la poca pulizia del mercurio, dopo alenne immersioni, sia pur delicate, quarzo e vetro si mostrano come lievenente appannati \& qualche gocciolina di mercurio comincia ad aderirvi. E allora questa « anormalità » del quarzo o del vetro sparisce per strofinio con la mano, col cotone, con la lana, insomma pulendo la superficie del dielettrico.

Ho già avuto occasione di accennare che le superfici di mercurio e di quarzo dalle quali ho dedotto le mie conclusioni, erano sempre terse.

Christiansen '), ha fatto numerose esperienze sulla elettrizzazione dovuta al contatto tra mercurio e isolanti, in

1) Wied. Ann., 53, pag. 401, 1894. 
partioolare tra mercurio e vetro. Fgli ha attribuito all'ossigeno i fenomeni osservati; ha considerato in particolare l'inflnenza dell' ossigeno adsorbito dagli isolanti; nel caso particolare del vetro trova delle variazioni di eccitabilità col tempo, che ricordano il fenomeno da mo descritto; ma, come auche it shaw, ha dato forse tropla importanza alle variazioni che avengono vellisolante, mentre che le esperiense da me descritte pongono fuori dubbio l'esistenza di una modificazione superyiciule del mercurio, di importanza eccezionale per Vellotizazione detta per strofinio.

\section{$\S 7$.}

Il fenomeno dhe ho pleso in esame è certamente particolare; ma io credo che prossa contribuire dal suo canto a far luce nelia questione ancon così complessa della elettrizzazione per strotinio.

Thtti sono dacordo nel ritenere che la cansa di questa. elettrizzazione sia nelle differenze fisiche tra le due superfici a contatto a sia dello stessia natura della causa producente l'elettriazame per confatto; ma finora si conoscolo" solo alcune rabaioni particolari e nou prive di eccezioni to." le proprieti fisiclue o lelettrizzazione per strofinio (legge di Coehn, leggi di Hesehus). Ciò è dovuto alla inesatta conoscenza delle condizioni superficiali dei dielettrici utilizzati, molti dei quali - e i più commi - sono corpi chimicamente non definiti e difficilmente riproducibili in condizioni fisiche sensibilmente ugnali. Per questa difficoltà, arevo iniziato le mie esperienze, dirette ad uno scopo molto generale (verifica della identità d'origine del fenomeno Volta e del fenomeno di elettrizzazione per strofinio in modo più completo di quanto facciano le esperieute di J. Thomson, di Hoorweg, di (hristianseu), sulla coppia mercurio-quarzo.

Come si i visto, anche in questo caso le esperienze avevano coudotto a risultati disparati; le mie ricerche hanno 
indicato un fatto nuovo, mediante il quale diventa possibile il coordinamento di tali risultati.

Gabinetto di Fisica del R. Liceo Alfleri Torino, 20 Febbraio 1920. 